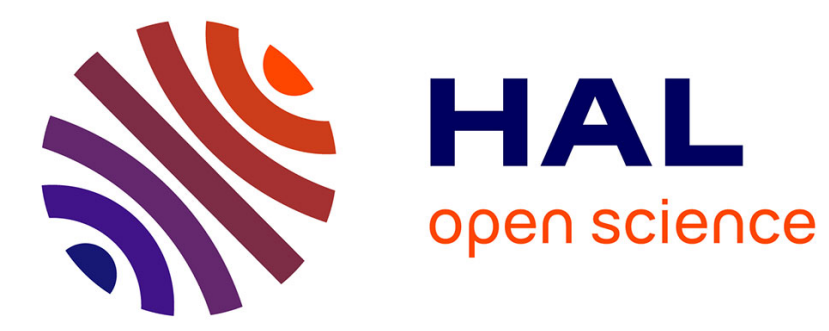

\title{
Influence of piezoelectricity on the photorefractive effect
} Gilles Pauliat, Pierre Mathey, Gérald Roosen

\section{To cite this version:}

Gilles Pauliat, Pierre Mathey, Gérald Roosen. Influence of piezoelectricity on the photorefractive effect. Journal of the Optical Society of America B, 1991, 8 (9), pp.1942-1946. hal-00856179

\section{HAL Id: hal-00856179 \\ https://hal-iogs.archives-ouvertes.fr/hal-00856179}

Submitted on 30 Aug 2013

HAL is a multi-disciplinary open access archive for the deposit and dissemination of scientific research documents, whether they are published or not. The documents may come from teaching and research institutions in France or abroad, or from public or private research centers.
L'archive ouverte pluridisciplinaire HAL, est destinée au dépôt et à la diffusion de documents scientifiques de niveau recherche, publiés ou non, émanant des établissements d'enseignement et de recherche français ou étrangers, des laboratoires publics ou privés. 


\title{
Influence of piezoelectricity on the photorefractive effect
}

\author{
G. Pauliat, P. Mathey, and G. Roosen \\ Institut d'Optique Théorique et Appliquée, Unité Associée au Centre National de la Recherche Scientifique, Centre \\ Scientifique d'Orsay, B.P. 147, 91403 Orsay Cedex, France
}

Received October 5, 1990; revised manuscript received February 2, 1991

\begin{abstract}
We demonstrate that the spatially modulated electric field that is associated with a photorefractive grating generates stress and strain components with symmetries that are different from those induced by a uniform electric field. Therefore, because of piezoelectricity and the elasto-optic effect, the symmetries of the effective dielectric and electro-optic constants to be used to describe the photorefractive effect differ from the symmetries of the usual dielectric and electro-optic tensors. We derive analytical expressions to be used to compute these new constants from the measured clamped and unclamped dielectric and electro-optic coefficients. Experimental evidence is presented.
\end{abstract}

\section{INTRODUCTION}

The physics of the photorefractive effect is now well understood. ${ }^{1}$ A photorefractive grating results from a photoinduced charge redistribution in the bulk of the material. The associated photoinduced electric field in turn generates an index change. To predict the kinetics and the steady state of the photorefractive effect, it is therefore necessary to determine the exact relations, first, between the charge pattern and the electric field and, second, between the electric field and the index grating. Because of the elasto-optic effect, the deformations induced by the electric field (piezoelectricity ${ }^{2}$ ) must be taken into account. For example, Stepanoy et $a l .{ }^{3}$ demonstrated the influence of piezoelectricity (by means of the elasto-optic effect) on the optical index changes in cubic crystals. The dielectric tensor and electro-optic tensor have already been measured for most photorefractive materials and for both clamped and unclamped samples. However, these results, obtained by applying a uniform electric field to the crystal, cannot be directly used for spatially modulated electric fields such as those associated with photorefractive gratings. Here we explain that the deformations induced by spatially modulated electric fields indeed necessarily differ from those induced by uniform fields. We demonstrate that the effective dielectric and electro-optic coefficients that should be used in photorefractive experiments are neither the clamped nor the unclamped coefficients determined by the usual measurements but that they can be computed from these values.

The notation that we need for the mathematical analysis is given in Section 2. In Section 3 we recall the wellknown results concerning the refractive index and dielectric changes induced by a uniform electric field. In Section 4 we explain the mechanism of the breaking of the crystal symmetry by a spatially modulated electric field. Then we derive the expressions for the effective dielectric and electro-optic coefficients, and we apply these results to $\mathrm{BaTiO}_{3}$ and $\mathrm{Bi}_{12} \mathrm{GeO}_{20}$ crystals.

\section{BASIC NOTATION}

An internal electric field $\mathbf{E}$ inside a piezoelectric crystal modifies the stress $\sigma$, the strain $\delta$, and the electric dis- placement D. We may choose at least two different equivalent sets of independent variables. ${ }^{2}$ In what follows we use $(\boldsymbol{\sigma}, \mathbf{E})$ and $(\boldsymbol{\delta}, \mathbf{E})$. With the Einstein summation convention we have

$$
\begin{aligned}
& \delta_{i j}=s_{i j k l} \sigma_{k l}+d_{k i j} E_{k}, \\
& D_{i}=d_{i j k} \sigma_{j k}+\epsilon_{0} \epsilon_{i j}^{\sigma=0} E_{j},
\end{aligned}
$$

or

$$
\begin{aligned}
& \sigma_{i j}=c_{i j k l} \delta_{k l}-e_{k i j} E_{k}, \\
& D_{i}=e_{i j k} \delta_{j k}+\epsilon_{0} \epsilon_{i j}^{\delta=0} E_{j},
\end{aligned}
$$

where

$\delta_{i j}, \sigma_{i j}, E_{i}$, and $D_{i}$ are the components of the strain tensor, stress tensor, electric field, and electric displacement;

$s_{i j k l}$ and $c_{i j k l}$ are the components of the elastic tensor and rigidity tensor;

$e_{i j k}$ and $d_{i j k}$ are the components of the piezoelectric tensors;

$\epsilon_{0}$ is the free-space permittivity, and $\epsilon_{i j}^{\delta=0}$ and $\epsilon_{i j}^{\sigma=0}$ are the components of the relative static dielectric tensors for totally clamped (strain-free) and totally unclamped (stressfree) crystals.

Equations (1) and (2) are equivalent, and the relations between their coefficients can be easily determined. ${ }^{2}$ Quite often Eqs. (1) and (2) are expressed with abbreviated notation for simplicity. However, this abbreviated notation is not convenient when changes of axes are needed, and therefore we use it not to carry out the calculation but only to give the numerical values of coefficients.

The effect of the internal electric field $\mathbf{E}$ and of the induced strain $\delta$ on the impermeability is given by the change in the impermeability tensor, which has components $\Delta \eta_{i j}$ :

$$
\Delta \eta_{i j}=r_{i j k}^{\delta=0} E_{k}+p_{i j k l}^{E=0} \delta_{k l},
$$

with $r_{i j k}^{\delta=0}$ the components of the totally clamped electrooptic tensor and $p_{i j k l}^{E=0}$ the components of the elasto-optic tensor. 
The nonvanishing values for the various coefficients of a number of crystals are listed in Appendix A by using abbreviated notations. ${ }^{2}$ The electro-optic coefficients given in Appendix A are the totally unclamped coefficients.

In what follows, given the electric field, we calculate the components $\Delta \eta_{i j}$ and $D_{i}$. We first determine the strain $\delta$.

\section{UNIFORM INTERNAL ELECTRIC FIELDS}

In this section we assume that a given voltage is applied to the sample, resulting in a uniform internal field. $d$ is the spacing between the two parallel electrodes, the modulus of the internal field is $V / d$, and its direction is perpendicular to the electrodes. We also assume that the strain and the stress are uniform, so that their values inside the material are equal to their values on the boundaries. If the sample is totally unclamped (e.g., $\sigma_{i j}=0$ ), the strain components induced by the electric field are given by Eq. (1a). Inserting their values into Eq. (2b) and comparing the result to Eq. (1b), we get

$$
\epsilon_{i j}^{\sigma=0}=\epsilon_{i j}^{\delta=0}+e_{i k l} d_{j k l} / \epsilon_{0} .
$$

Similarly, we derive the expression for the totally unclamped electro-optic coefficients $r_{i j k}^{\sigma=0}$ by using Eq. (3):

$$
r_{i j k}^{\sigma=0}=r_{i j k}^{\delta=0}+p_{i j l m} d_{k l m} .
$$

We note that the totally clamped coefficients given in Eqs. (4) and (5) do not correspond to the so-called clamped coefficients that are measured in some experiments where only the deformation along the field axis is forbidden; in the above expressions all six possible deformations are forbidden. We computed the differences between strain-free and stress-free coefficients according to the values listed in Appendix A.

We found that for $\mathrm{BaTiO}_{3}$

$$
\epsilon_{1}^{\sigma=0}-\epsilon_{1}^{\delta=0}=2600, \quad \epsilon_{3}^{\sigma=0}-\epsilon_{3}^{\delta=0}=45,
$$

for $\mathrm{Bi}_{12} \mathrm{GeO}_{20}$

$$
\epsilon_{1}^{\sigma=0}-\epsilon_{1}^{\delta=0}=4.3, \quad r_{41}^{\sigma=0}-r_{41}^{\delta=0}=0.34 \mathrm{pm} / \mathrm{V},
$$

and for $\mathrm{GaAs}$

$$
\epsilon_{1}^{\sigma=0}-\epsilon_{1}^{\delta=0}=5 \times 10^{-2}, \quad r_{41}^{\sigma=0}-r_{41}^{\delta=0}=0.19 \mathrm{pm} / \mathrm{V} .
$$

\section{SPATIALLY MODULATED INTERNAL ELECTRIC FIELDS}

\section{A. Determination of the Strain Components}

The above analysis is no longer possible when the electric field is nonuniform. Equations (1) and (2) are not sufficient to determine the deformation at every location inside the material. They give only a relation between the components of $\delta, \sigma, \mathbf{E}$, and $\mathbf{D}$. For example, in a cubic material (23 or $\overline{4} 3 \mathrm{~m}$ ), a simple (and wrong) analysis of the symmetries (see Appendix A) seems to indicate that if a field grating is along the $x$ crystallographic axis then only $\delta_{y z}, \delta_{z y}, \sigma_{y z}$, and $\sigma_{z y}$ can be different from zero. Actually shear deformations (induced by $\delta_{y z}$ and $\delta_{z y}$ ) are spatially modulated along the same axis as the internal field. The macroscopic deformation ${ }^{2}$ along the $z$ axis being

$$
U_{z}=\int \delta_{z x} \mathrm{~d} x+\delta_{z y} \mathrm{~d} y+\delta_{z z} \mathrm{~d} z
$$

the strain components $\delta_{z x}$ and $\delta_{x z}$, equal to $\partial U_{z} / \partial x$, are therefore also not equal to zero (as is true for many others). The relations among $\delta_{x z}, \delta_{z x}, \sigma_{x z}$, and $\sigma_{x y}$ are still given by Eqs. (1a) and (1b). This nonlocal effect is general for all point groups and cannot be predicted from Eqs. (1) and (2) alone.

To complete Eqs. (1) or (2), we can use the first principle of dynamics. ${ }^{4}$ The forces that are due to stress vanish if the sample is at equilibrium. For a unit volume the expressions of their components are ${ }^{2}$

$$
F_{i}=\frac{\partial \sigma_{i j}}{\partial x_{j}}=0
$$

The boundary conditions impose the values of $\sigma_{i j}$ on the crystal faces. Equation (6) is valid as soon as the photorefractive buildup time constant $\tau_{g}$ is much larger than the characteristic time constant of the mechanical vibration. If $\Lambda$ is the grating fringe spacing and if $v$ is the velocity of sound, then this condition can be expressed by

$$
\tau_{g} \gg \Lambda / v .
$$

The velocity of sound being equal to a few kilometers per second and the fringe spacing being equal to a few micrometers, $\tau_{g}$ must be greater than a few nanoseconds. Therefore condition (7) is fulfilled in most experiments.

We must remark that, even in the absence of external forces, Eq. (6) does not imply that the stress components $\sigma_{i j}$ are equal to zero inside the sample. In the more general case strain and stress depend on the three space coordinates. We now restrict our analysis to quasi-infinite crystals whose dimensions are much larger than the fringe spacing $\Lambda$ of the induced grating. This situation corresponds to most photorefractive experiments, in which the fringe spacing is a few micrometers and the sample dimensions are a few millimeters. However, our analysis does not apply to thin samples, where surface deformations are of importance. ${ }^{5}$ For infinite samples the symmetry implies that the deformation is possible only along the grating wave vector, ${ }^{6}$ which is also the field axis. We thus conduct the calculation for a set of axes in which the first axis $x^{\prime}$ is parallel to the electric field. All the components in this new set are denoted by a prime. The space derivatives with respect to $y^{\prime}$ and $z^{\prime}$ vanish, and Eq. (6) reduces to

$$
\frac{\partial \sigma_{x^{\prime} x^{\prime}}^{\prime}}{\partial x^{\prime}}=0 .
$$

As a consequence, the modulated part of $\sigma^{\prime} x^{\prime} x^{\prime}$ equals zero. From Eq. (2) we immediately obtain the only nonzero strain component $\delta_{x^{\prime} x^{\prime}}^{\prime}$ :

$$
\begin{aligned}
& \delta_{x^{\prime} x^{\prime}}^{\prime}=\frac{e_{x^{\prime} x^{\prime} x^{\prime}}^{\prime}}{c_{x^{\prime} x^{\prime} x^{\prime} x^{\prime}}^{\prime}} E, \\
& \delta_{x^{\prime} y^{\prime}}^{\prime}=\delta_{x^{\prime} z^{\prime}}^{\prime}=\delta_{y^{\prime} z^{\prime}}^{\prime}=\delta_{y^{\prime} y^{\prime}}^{\prime}=\delta_{z^{\prime} z^{\prime}}^{\prime}=0,
\end{aligned}
$$

where $E$ denotes the modulus of the field $\mathbf{E}$.

If $a_{i}$ denotes the direction cosines of the electric field $\mathbf{E}$ in the crystallographic axes, then $\delta^{\prime}{ }_{x^{\prime} x^{\prime}}$ is expressed as a 


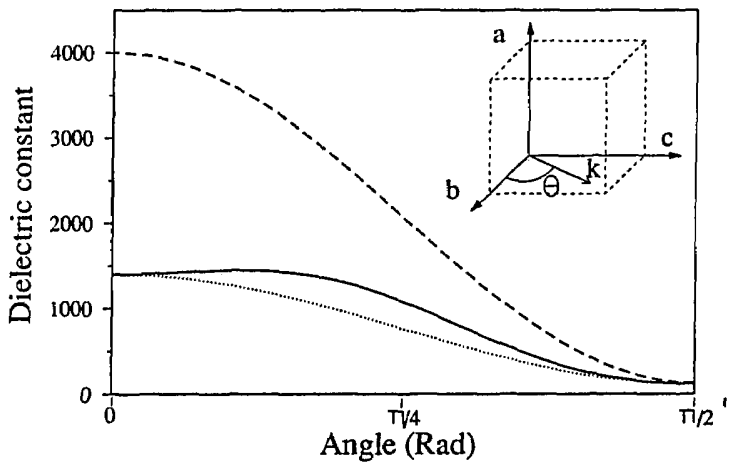

Fig. 1. Effective dielectric constant for $\mathrm{BaTiO}_{3}$ versus the angle $\theta$ between the $b$ crystallographic axis and the direction $k$ of the internal electric field, $k$ being in the $(b, c)$ plane: solid curve, spatially modulated field; dashed curve, uniform field and stressfree sample; dotted curve, uniform field and strain-free sample.

function of the piezoelectric and rigidity tensor components given in those axes:

$$
\delta_{x^{\prime} x^{\prime}}^{\prime}=H E,
$$

with

$$
H=\frac{\sum_{i, j, k=1}^{3} a_{i} a_{j} a_{k} e_{i j k}}{\sum_{l, m, n, o=1}^{3} a_{l} a_{m} a_{n} a_{o} c_{l m n o}} .
$$

The strain components can also be expressed relative to the crystallographic axes:

$$
\delta_{i j}=a_{i} a_{j} H E .
$$

Equation (12) demonstrates that a photorefractive grating can generate strain components that cannot be induced by a uniform electric field (and conversely a uniform field can induce strain components that cannot be created by a photorefractive grating). For instance, for cubic crystals and according to Eq. (1a), with the $d_{i j k}$ coefficients given in Appendix A, a spatially uniform electric field along the [111] crystallographic direction can induce only shear deformations $\left(\delta_{i j}\right.$ with $\left.i \neq j\right)$. However, if the field is spatially modulated then all the strain components are equal and differ from zero [Eq. (12)].

We can thus conclude that the piezoelectric tensor symmetry is modified by the inhomogeneous field.

\section{B. Determination of the Effective Dielectric Constant}

We derive the relative dielectric tensor $\epsilon^{\mathrm{pr}}$, to be used when the electric field is spatially modulated, by inserting Eq. (12) for $\delta_{i j}$ into Eq. (2b). We get

$$
\epsilon_{i j}^{p r}=\epsilon_{i j}^{\delta=0}+e_{i k j} a_{k} \frac{\mathbf{H}}{\epsilon_{0}} .
$$

Equation (13) is not convenient for performing the calculation and can be misleading because $\epsilon^{\mathrm{pr}}$ itself depends on the electric field by means of its direction cosines. Poisson's law, which links the modulated electric charge grating to the electric-field displacement, is used to define an effective photorefractive static dielectric constant $\epsilon_{\text {eff }}^{\text {pr }}$ :

$$
\operatorname{div}\left(\epsilon^{\mathrm{pr}} \mathbf{E}\right)=\epsilon_{\mathrm{eff}}^{\mathrm{pr}} \operatorname{div} \mathbf{E} .
$$

From Eqs. (13) and (14) we immediately get

$$
\epsilon_{\mathrm{eff}}^{\mathrm{pr}}=a_{i}\left(\epsilon_{i j}^{\delta=0}+e_{i k j} a_{k} \frac{\mathbf{H}}{\epsilon_{0}}\right) a_{j} .
$$

\section{Effective Electro-optic Coefficient}

In the crystallographic axes the coupling of two optical waves incident upon a photorefractive grating is related to the nonlinear dielectric tensor for optical frequencies:

$$
\Delta \epsilon_{i j}^{\omega}=-n_{i}{ }^{2} n_{j}{ }^{2}\left(\Delta \eta_{i j} / \epsilon_{0}\right)
$$

where the values $n_{i}{ }^{2}=1 / \varepsilon_{i i}{ }^{\omega}$ are the squares of the optical refractive indices.

$v_{i}$ and $w_{i}$ being the components of the two optical wave polarization vectors, the coupling strength is proportional
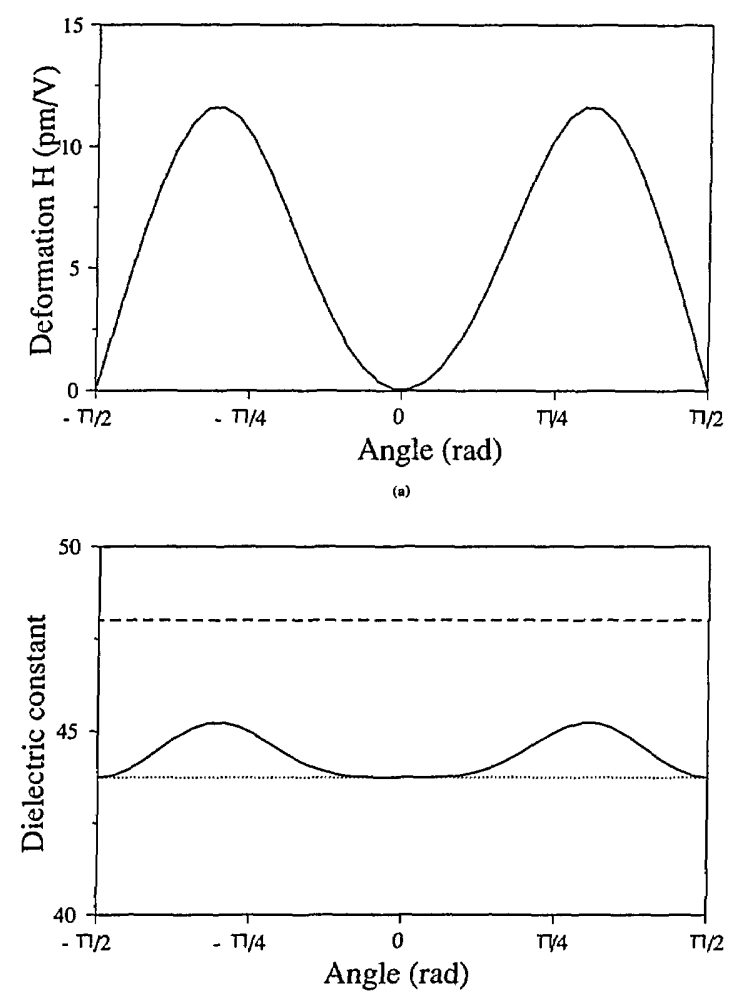

(b)

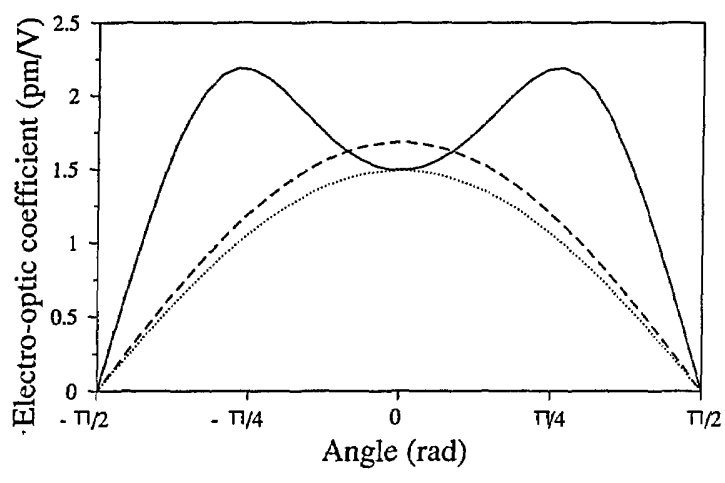

(c)

Fig. 2. Dependence of the various coefficients for $\mathrm{Bi}_{12} \mathrm{GeO}_{20}$ on the angle $\theta$ between the [001] axis and the direction $k$ of the internal electric field, $k$ being in the (110) plane. (a) Deformation $H$ as defined by Eq. (11). (b) Effective dielectric constant. (c) Ratio $r_{\text {eff }} / n^{4}$ : solid curve, spatially modulated field; dashed line, uniform field and stress-free sample; dotted line, uniform field and strain-free sample. 


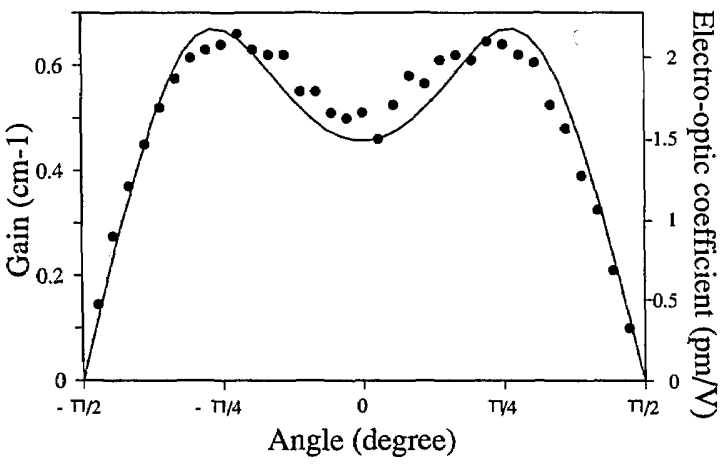

Fig. 3. Experimental data of two-beam-coupling gain (dots) and theoretical plot of the variation (solid curve) of the effective electro-optic coefficient $r_{\text {eff }} / n^{4}$ versus angle $\theta$, with the same configuration as in Fig. 2 in the case of a modulated field. The sample is a $\mathrm{Bi}_{12} \mathrm{GeO}_{20}$ crystal, and we used the numerical values given in Appendix A for that type of material.

to the effective electo-optic coefficient $r_{\text {eff }}^{\text {pr }}$ :

$$
r_{\mathrm{eff}}^{\mathrm{pr}}=-v_{i}^{*} \frac{\epsilon_{0} \Delta \epsilon_{i j}}{E} w_{j}
$$

which can be rewritten by using Eqs. (3) and (12):

$$
r_{\mathrm{eff}}^{\mathrm{pr}}=v_{i}^{*}\left(r_{i j k}^{\delta=0}+p_{i j l k} a_{l} H\right) a_{k} w_{j} n_{i}^{2} n_{j}^{2} .
$$

The asterisk denotes complex conjugation.

Equations (15) and (18) show that the constants that are valid for a description. of the photorefractive effect are neither the clamped nor the unclamped ones. According to Eq. (8), the crystal must indeed always be considered as internally unclamped along the field axis $x^{\prime}$, whatever the applied external forces are. Conversely, the other stress components may be different from zero. Note that the breaking of the crystal symmetry by the spatially modulated field permits a nonzero $r_{\text {eff }}^{\mathrm{pr}}$ coefficient [Eq. (18)] in a configuration (a given field direction and given optical beam polarizations) for which a uniform field [Eq. (5)] would not induce any index change. For example, for a crystal belonging to the point-group symmetry $\overline{4} 3 \mathrm{~m}$, a modulated field grating along the [111] crystallographic direction can induce an isotropic coupling between two optical waves polarized along one crystallographic axis. Such an isotropic coupling in these materials is forbidden by the usual symmetry of the electro-optic tensor $\left(r_{i j}=0\right)$.

\section{Application to $\mathrm{BaTiO}_{3}$}

Experiments are usually performed in $\mathrm{BaTiO}_{3}$ samples with the induced grating wave vector lying in the $(b, c)$ crystallographic plane. In Fig. 1 we have plotted the dielectric constant $\epsilon_{\mathrm{eff}}^{\mathrm{pr}}$, given by Eq. (15), versus the angle $\theta$ between the grating wave vector and the crystallographic $b$ axis. For comparison we have also plotted the effective dielectric constants for a uniform electric field and for totally unclamped (dashed curve) and clamped (dotted curve) crystals. These constants are defined as $\epsilon_{\mathrm{eff}}^{\mathrm{pr}}$ by Eq. (15) with $\epsilon_{i j}^{g=0}$ or $\epsilon_{i j}^{\delta=0}$ substituted for $\epsilon_{i j}^{\mathrm{pr}}$.

Figure 1 shows that the value of the effective dielectric constant that is to be used with a modulated field and in this particular crystallographic orientation lies between the clamped and unclamped values. Unfortunately, we could not find any values for the elasto-optic coefficients in the literature, and therefore we cannot present any conclusion for the effective electro-optic coefficient.

\section{E. Application to $\mathrm{Bi}_{12} \mathrm{GeO}_{20}$}

We consider the special case of a grating with a wave vector lying in the (110) plane, and we study the variation of both $\varepsilon_{\text {eff }}^{\mathrm{pr}}$ and $r_{\mathrm{eff}}^{\mathrm{pr}}$ as a function of the angle $\theta$ between the [001] axis and the wave vector. In Fig. 2(a) the coefficient $H$ (e.g., the deformation) is plotted versus $\theta$. $H$ shows a maximum for $\theta= \pm 55^{\circ}$. Figure 2(b) shows the effective dielectric constant for a spatially modulated electric field (solid curve) and for a uniform field for both totally unclamped (dashed line) and clamped (dotted line) samples. In Fig. 2(c) we have plotted the ratio $r_{\mathrm{eff}}^{\mathrm{pr}} / n^{4}$ (solid curve) seen by two circularly polarized interfering beams with an external incident angle equal to $\pm 15^{\circ}$. For comparison we have also plotted the effective coefficients by using the totally unclamped and clamped electro-optic tensors in place of the term in parentheses in Eq. (18).

For $\theta=0^{\circ}, r_{\mathrm{eff}}^{\mathrm{pr}}$ is equal to the clamped value [no deformation as seen in Fig. 2(b)]. However, for $\theta \approx \pm 45^{\circ} r_{\mathrm{eff}}^{\mathrm{pr}}$ presents two maxima that are larger than both the clamped and unclamped values. For this orientation (electric field along the [111] crystallographic direction) all possible deformations are excited, and they produce the strongest elasto-optic index change. These two maxima were observed by Stepanov et al. ${ }^{3}$ The maxima have equal magnitude because we assume a symmetric elastooptic tensor: $\quad p_{i j k l}=p_{k l i j}$. However, that assumption is not necessarily valid for materials that belong to the point-group symmetry 23 , so that the two maxima could be of unequal magnitudes.

To check these predictions experimentally, we conducted a two-beam-coupling experiment with a $\mathrm{Bi}_{12} \mathrm{GeO}_{20}$ sample in the configuration described above. Two circularly polarized beams from an $\mathrm{Ar}^{+}$laser $(514 \mathrm{~nm}$ ) wrote a grating with a fringe spacing of $1 \mu \mathrm{m}$. The experimental values for the two-wave mixing gain, proportional to $r_{\text {eff }}^{\mathrm{pr}}$, are reported in Fig. 3. The experimental data are in good agreement with the theoretical behavior predicted by Eq. (18).

\section{CONCLUSIONS}

Piezoelectricity has a large influence on the dielectric and piezoelectric constants in photorefractive materials. In the presence of a uniform electric field these constants depend on externally applied forces. However, those to be used for a spatially modulated field are independent of external forces. Therefore, if one is to describe the photorefractive effect in the presence of an externally applied voltage, different values must be used simultaneously for the dielectric and the electro-optic constants, the first values for the uniform part of the field and the others for its modulated component. We have demonstrated that, because of the breaking of the crystal symmetry by the internal field, the symmetry of the effective electro-optic coefficient is modified in the presence of a photorefractive charge grating. This effective coefficient can be either larger or smaller than both the strain-free (totally clamped) or stress-free (totally unclamped) electrooptic coefficients. The coefficient can also be different from zero in a configuration in which both the clamped and unclamped coefficients vanish. 


\section{APPENDIX A}

Here nonvanishing coefficients for some photorefractive materials are expressed with abbreviated notations. ${ }^{2}$

The pairs of indices $i, j$ and $k, l(i, j, k$, and $l$ from 1 to 3$)$ are contracted to $m$ and $n$, which take on values from 1 to 6 according to

$$
\begin{gathered}
11 \rightarrow 1, \quad 22 \rightarrow 2, \quad 33 \rightarrow 3, \quad 23 \rightarrow 4, \quad 32 \rightarrow 4, \\
13 \rightarrow 5, \quad 31 \rightarrow 5, \quad 23 \rightarrow 6, \quad 32 \rightarrow 6 .
\end{gathered}
$$

In contrast to tensors $\mathbf{s}$ and $\mathbf{d}$, for those components given below no multiplying factor must be introduced between the full and abbreviated notations, so that for any $i, j, k$, or $l$ we always have

$$
\begin{gathered}
c_{i j k l}=c_{m n}, \quad \epsilon_{i j}=\epsilon_{m}, \quad e_{i k l}=e_{i m}, \\
r_{i j k}=r_{m k}, \quad p_{i j k l}=p_{m n} .
\end{gathered}
$$

$\mathrm{BaTiO}_{3}$, Point-Group Symmetry $4 \mathrm{~mm}$

Rigidity tensor ${ }^{7} c_{i j}=c_{j i}\left(10^{10} \mathrm{~N} / \mathrm{m}^{2}\right)$ :

$$
\begin{gathered}
c_{11}=c_{22}=24.5, \quad c_{12}=12.8, \quad c_{13}=12.3, \\
c_{33}=14.8, \quad c_{44}=c_{55}=5.5, \quad c_{66}=12.0 .
\end{gathered}
$$

Stress-free dielectric constant ${ }^{\text {? }}$ :

$$
\epsilon_{1}=\epsilon_{2}=4000, \quad \epsilon_{3}=130 .
$$

Piezoelectric tensor ${ }^{7}\left(\mathrm{C} / \mathrm{m}^{2}\right)$ :

$$
e_{15}=e_{24}=35.6, \quad e_{31}=e_{32}=-3.96, \quad e_{33}=1.92 .
$$

Stress-free electro-optic tensor ${ }^{8,9}(\mathrm{pm} / \mathrm{V})$ :

$$
r_{13}=r_{23}=19.5, \quad r_{33}=97, \quad r_{42}=1640 .
$$

GaAs, Point-Group Symmetry $\overline{\mathbf{4}} 3 \mathrm{~m}$

Rigidity tensor ${ }^{10} c_{i j}=c_{j i}\left(10^{10} \mathrm{~N} / \mathrm{m}^{2}\right)$ :

$$
\begin{gathered}
c_{11}=c_{22}=c_{33}=11.90, \quad c_{12}=c_{13}=c_{23}=5.38, \\
c_{44}=c_{55}=c_{66}=5.95 .
\end{gathered}
$$

Stress-free dielectric ${ }^{10}$ constant at $T=300 \mathrm{~K}$ :

$$
\epsilon_{1}=\epsilon_{2}=\epsilon_{3}=12.85 \text {. }
$$

Piezoelectric tensor ${ }^{\mathrm{t0}}\left(\mathrm{C} / \mathrm{m}^{2}\right)$ :

$$
e_{14}=e_{15}=e_{36}=0.154 \text {. }
$$

Stress-free electro-optic tensor ${ }^{11}(\mathrm{pm} / \mathrm{V})$ :

$$
r_{41}=r_{52}=r_{63}=1.2 \text {. }
$$

Elasto-optic tensor ${ }^{12}\left(p_{i j}=p_{j i}\right)$ :

$$
\begin{gathered}
p_{11}=p_{22}=p_{33}=0.165, \quad p_{12}=p_{13}=p_{23}=0.14, \\
p_{44}=p_{65}=p_{66}=0.072 .
\end{gathered}
$$

$\mathrm{Bi}_{12} \mathrm{GeO}_{20}$, Point-Group Symmetry 23

Rigidity tensor ${ }^{13} c_{i j}=c_{j i}\left(10^{10} \mathrm{~N} / \mathrm{m}^{2}\right)$ :

$$
\begin{gathered}
c_{11}=c_{22}=c_{33}=12.8, \quad c_{12}=c_{13}=c_{23}=3.05, \\
c_{44}=c_{65}=c_{66}=2.55 .
\end{gathered}
$$

Stress-free dielectric constant ${ }^{14}$ :

$$
\epsilon_{1}=\epsilon_{2}=\epsilon_{3}=48
$$

Piezoelectric tensor ${ }^{13}\left(\mathrm{C} / \mathrm{m}^{2}\right)$ :

$$
e_{14}=e_{15}=e_{36}=0.98 \text {. }
$$

Stress-free electro-optic tensor ${ }^{15}(\mathrm{pm} / \mathrm{V})$ :

$$
r_{41}=r_{52}=r_{63}=3.6 \text {. }
$$

Elasto-optic tensor ${ }^{3}\left(p_{i j} \neq p_{j i}\right)$ :

$$
\begin{gathered}
p_{11}=p_{22}=p_{33}=0.12, \quad p_{12}=p_{13}=p_{23}, \\
p_{21}=p_{31}=p_{32},
\end{gathered}
$$

with

$$
p_{12}+p_{13}=0.19, \quad p_{44}=p_{55}=p_{66}=0.01 .
$$

\section{ACKNOWLEDGMENT}

We are greatly indebted to J. C. Launay (Université de Bordeaux, France) for providing the $\mathrm{Bi}_{12} \mathrm{GeO}_{20}$ sample.

\section{REFERENCES}

1. P. Günter and J. P. Huignard, eds., Photorefractive Materials and Their Applications I, Vol. 62 of Topics in Applied Physics (Springer-Verlag, Berlin, 1989).

2. J. F. Nye, Physical Properties of Crystals (Clarendon, Oxford, 1957).

3. S. I. Stepanov, S. M. Shandarov, and N. D. Khat'kov, "Photoelastic contribution to the photorefractive effect in cubic crystals," Sov. Phys. Solid State 29, 1754 (1987).

4. L. D. Landau and E. M. Lifchitz, eds., Théorie de lélasticité, (Mir, Moscow, 1967).

5. A. M. Bliznetsov, M. P. Petrov, and A. V. Khomenko, "Photoinduced piezoelectric phase modulation of light by crystals," Sov. Tech. Phys. Lett. 10, 463 (1984).

6. D. W. Pohl, "A note on the polarization of light scattered from entropy and LA fluctuations in solids," Solid State Commun. 23, 447 (1977).

7. A. Schaefer, H. Schmitt, and A. Dörr, "Elastic and piezoelectric coefficients of TSSG barium titanate single crystals," Ferroelectrics 69, 253 (1986).

8. A. R. Johnston and J. M. Weingart, "Determination of the low-frequency linear electro-optic effect in tetragonal $\mathrm{BaTiO}_{3}$," J. Opt. Soc. Am. 55, 828 (1965).

9. S. Ducharme, J. Feinberg, and R. R. Neurgaonkar, "Electronic and piezoelectric measurements in photorefractive barium titanate and strontium barium niobate," IEEE J. Quantum Electron. QE-23, 2116 (1987).

10. J. S. Blakemore, "Semiconducting and other major properties of gallium arsenide," J. Appl. Phys. 53, R123 (1982).

11. M. Sugie and $K$. Tada, "Measurements of the linear electrooptic coefficients and analysis of the nonlinear susceptibilities in cubic GaAs and hexagonal CdS," Jpn. J. Appl. Phys. 15,421 (1976).

12. K.-H. Hellwege, ed., Landolt-Börnstein, Numerical Data and Functional Relationships in Science and Technology. Group III: Crystal and Solid State Physics (SpringerVerlag, Berlin, 1969).

13. A. J. Slobodnik, Jr., and J. C. Sethares, "Elastic, piezoelectric, and dielectric constants of $\mathrm{Bi}_{12} \mathrm{GeO}_{20}$," J. Appl. Phys. 43, 247 (1972).

14. F. Marsaud, "Croissance par la méthode de Czochralski de monocristaux de $\mathrm{Bi}_{12} \mathrm{GeO}_{20}$ purs et dopés, caractérisations physico-chimiques et optimisation de ses propriétés photoréfractives," D.S. dissertation, order no. 155 (Université de Bordeaux I, Talence, France, 1987).

15. V. P. Lenzo, E. G. Spencer, and A. A. Ballman, "Optical activity and electro-optic effect in bismuth germanium oxide $\mathrm{Bi}_{12} \mathrm{GeO}_{20}$," Appl. Opt. 5, 1688 (1966). 